\title{
Author Correction: Associated and Mediating Variables Related to Job Satisfaction among Professionals from Mental Health Teams
}

\author{
Marie-Josée Fleury ${ }^{1,2}$ • Guy Grenier ${ }^{2}$ • \\ Jean-Marie Bamvita ${ }^{2}$ - François Chiocchio ${ }^{3}$
}

C) Springer Science+Business Media, LLC, part of Springer Nature 2017

\section{Author Correction: Psychiatr Q \\ https://doi.org/10.1007/s11126-017-9543-6}

The original version of this article unfortunately contained a mistake in the author group section. The family name of Dr. François should be "Chiocchio" not "Chiochio."

The authors apologize to readers for this error.

The original article has been corrected.

The online version of the original article can be found at https://doi.org/10.1007/s11126-017-9543-6

\section{Marie-Josée Fleury}

flemar@douglas.mcgill.ca

1 Department of Psychiatry, McGill University, Montreal, QC, Canada

2 Douglas Hospital Research Centre, Douglas Mental Health University Institute Research Centre, 6875 LaSalle Blvd, Montreal, QC H4H 1R3, Canada

3 Telfer School of Management, University of Ottawa, 55 Laurier Avenue East, Ottawa, ON K1N 6N5, Canada 